\title{
Extended culture up to the blastocyst stage: a strategy to avoid multiple pregnancies in assisted reproductive technologies
}

\author{
Soledad J Sepúlveda, Jimmy R Portella, Luis P Noriega, Ernesto L Escudero \& Luis H Noriega \\ PRANOR Assisted Reproduction Group.
}

\begin{abstract}
The aim of this study was to review the experience and outcomes of assisted reproduction cycles with embryos grown up to day 5 of development, comparing different parameters according to the ages of the patients.

We retrospectively studied 1,874 assisted reproduction cycles where embryo culture was extended up to the fifth or sixth day of development. All IVF and ICSI cycles were included, comparing, according to patient age, the following rates: blastocyst formation, pregnancy, implantation and abortion. As control, we analyzed cycles with donated oocytes from young donors (OD).

The number of embryos reaching the blastocyst stage is similar in all groups of patients. Only the OD group was different in terms of blastocyst formation, pregnancy and implantation rates. Patients over 39 years of age had an abortion rate of $59.1 \%$, which is significantly higher than the other groups.

Extended embryo culture up to the blastocyst stage can be implemented in programs of assisted reproduction in order to increase the pregnancy rate. The potential of blastocyst implantation is high, allowing us to transfer fewer embryos and reduce the probability of multiple pregnancies.
\end{abstract}

Key words: blastocyst, long-term culture, pregnancy rate, implantation rate.

\section{INTRODUCTION}

The challenge for assisted reproductive techniques (ART) is to avoid multiple pregnancies, which could be caused by the high number of transferred embryos. Therefore, the goal is hopefully to transfer only one embryo, but well selected. In general, assisted reproduction laboratories select embryos to be transferred based on morphology, since it is the only simple tool. However, good morphology is not always related to the outcome of the embryo and may involve multiple aneuploidy.

In the last decade, culture systems have been developed that allow extended development up to the blastocyst stage and thereafter selection of the best embryos to be transferred (Gardner et al., 1998; Blake et al., 2007). However, early human development is inefficient and only a few zygotes will reach blastocyst stage and implantation. Therefore, an embryo that develops to the blastocyst stage undergoes spontaneous selection during in vitro culture.

\section{BLASTOCYST FORMATION}

After passing through the tube, the embryo is a morula. In peripheral blastomeres, tight junctions are established that seal the space among the outer cells that form trophoblasts. Sealing prevents the diffusion of intercellular material in the extraembryonic space and so the blastocoel cavity is formed. The morula, composed of a single-cell type, is transformed into a blastocyst, where two-cell types can be recognized. The peripheral blastomeres form a flattened epithelium called trophoblast, and central blastomeres form the inner cell mass. These cells give rise to the embryo itself and part of the embryonic annexes. The trophoblast gives rise to most of the annexes. The expanded human blastocyst contains over
90 cells, of which an estimated $10 \%$ form the inner cell mass (figure 1) (Sepúlveda, 2008).

The blastocoel cavity is formed by the confluence of vacuoles. It grows by the action of $\mathrm{Na}^{+} / \mathrm{K}^{+}$ATPase located in the membrane of trophoblast cells. $\mathrm{Na}+$ ions enter the cavity, which begins to incorporate water to maintain osmotic balance. Water movement during cavitation may also be facilitated by aquaporins in the apical and basolateral membranes in the trophectoderm (Barcroft et al., 2003). Thus, the cavity expands and begins hatching, after which the embryo adheres to the surface of the endometrium, invading and making contact with the mother's vascular system during implantation. This procedure can take a couple of days and goes through the stages illustrated in Figure 1. First, an early blastocyst is formed, with a small cavity, which grows to form a full blastocyst. From this stage, we begin to categorize the number of cells in the trophoblast and inner cell mass. Expansion begins and finally hatching through the zona pellucida (Figure 1). This classification allows selecting blastocysts for transfer that have higher growth potential. The logic behind extending embryo culture until the fifth day is not to improve its quality, but to increase the probability of choosing the embryo with better implantation potential.

\section{ADVANTAGES OF EXTENDED CULTURE TO BLASTOCYST}

It is important to have an embryo screening method that allows transferring the minimum number of embryos without affecting the chance of pregnancy. The development of media by which the embryos grow for longer periods, up to the blastocyst stage allows selecting the best embryos to be transferred. Thus, it has increased implantation rates and reduced multiple gestations. A meta-analysis, which evaluated 
18 randomized controlled studies, reported that there is a significant difference in rates of pregnancy and live birth deliveries for transfers on day 5 compared to transfers on day 3 (Blake et al., 2007).

The idea that sequential media are required for optimal embryo development has an intuitive appeal, but it is not supported by direct experimental evidence (Biggers et al., 2005). In such sequential media culture systems, the medium used for culture embryos from day 1 to 3 of development differs in composition and/or concentration of components from the medium used for subsequent culture from Day 3 to the blastocyst stage (Gardner \& Lane, 1997, 1998). We demonstrated that the in-vitro development to the blastocyst stage and subsequent implantation rates are better for human embryos cultured in a single medium than for those cultured in a sequential media system (Sepúlveda et al., 2009). Fertilization does not occur at the same time in all eggs, thus the timing of each embryo is different. Therefore a single medium would be better, where the embryo uses what it needs.

There are several advantages for the culture up to the blastocyst stage. As discussed earlier, the longer-term culture allows us to distinguish between viable embryos with the greatest potential for implantation from those that developed initially but die later. It also allows evaluating genomic activation of the embryo (Braude et al., 1988), looking for the

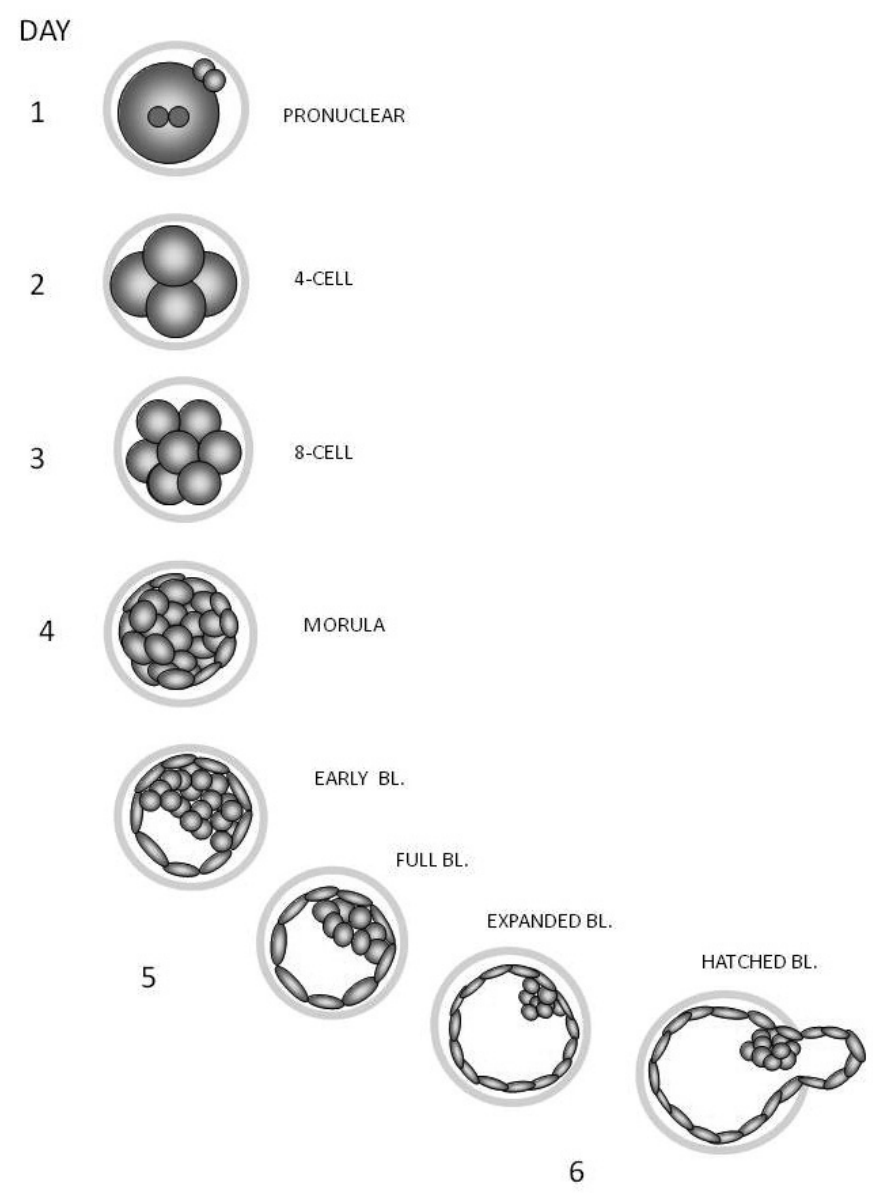

Figure 1: Human preimplantation development, since pronuclear stage on day 1 , until hatched blastocyst on day 6 . embryos that do not reach the 8-cell stage. Evidence shows that there is a lower rate of early abortion when blastocysts are transferred (Papanikolaou et al., 2006).

In vivo, cleavage stage embryos are usually in the fallopian tubes, not in the uterus, which is why synchronization of the transfer of embryos in the blastocyst stage with the uterine environment is more physiological (Gardner et al., 1996). Another advantage of long-term culture is the extended time, which allows for transfer when blastomeres are removed on day 3 for preimplantation genetic diagnosis.

The aim of this study was to review the experience and outcomes of assisted reproduction cycles with embryos at day 5 of development, comparing various parameters according to the age of the patient.

\section{MATERIALS AND METHODS}

We retrospectively studied 1,874 assisted reproductive cycles where embryos were cultured up to the fifth or sixth day of development, including procedures of oocyte donation (OD, $\mathrm{n}=1,192 ; 2004-2010)$, in vitro fertilization (IVF) and intracytoplasmic sperm injection (ICSI) $(n=682,2008-2010)$.

The following rates were compared according to the age of the patient: blastocyst formation, pregnancy, implantation and abortion.

\section{Insemination / injection}

For insemination in conventional IVF, about 100,000 sperm were co-cultured with cumulus-corona-oocyte complexes. For ICSI, only the metaphase II (MII) oocytes were injected, using procedures described earlier (Sepúlveda et al., 2009).

\section{Embryo culture}

Fertilized oocytes (2PN) were cultured individually in drops of $10 \mu \mathrm{L}$ Global ${ }^{\circledR}$ media (Global Life, Canada) supplemented with $10 \%$ synthetic serum substitute (SSS). On Day 3, embryos were changed to fresh Global® medium. Culture were under mineral oil at $37{ }^{\circ} \mathrm{C}$ in an atmosphere of $6.5 \% \mathrm{CO}_{2}, 5 \% \mathrm{O}_{2}$ and $88.5 \% \mathrm{~N}_{2}$. Assessments of embryonic development were conducted under the described procedures (Sepúlveda et al., 2009).

Embryo transfer

Embryo transfers were performed on day 5 or 6 of embryo culture, using a Frydman Ultrasoft catheter (CCD Laboratories, France). One or two embryos were selected for transfer. If more embryos were available, they were frozen or vitrified.

\section{Definition of analyzed parameters}

The blastocyst formation rate is the total number of blastocysts among the total 2PN oocytes. The pregnancy rate was defined as the number of cycles with at least a gestational sac among the number of cycles transferred. The abortion rate was defined as the number of pregnancies with total loss of gestational sacs before 20 weeks of gestation among the number of pregnancies. The implantation rate was calculated based on the number of gestational sacs among the total number of embryos transferred. 
Statistical analyses were performed using the STATA 10.0 statistical package (StataCorp LP, 4905 Lakeway Drive, College Station, TX, USA). The normality of distribution of continuous variables was assessed with a Kolmogorov-Smirnov test. Continuous variables were assessed with parametric statistic (Student's $t$-test) or non parametric statistics (Mann-Whitney Rank Sum Test). For non-continuous variables, the chisquare test or Fisher's exact test was used. Differences were considered significant with a p-value of $<0.05$.

\section{RESULTS AND DISCUSSION}

In the oocyte donation program, the blastocyst cultures began in 2004 with $4 \%$ of cycles. Currently, the extended culture up to the blastocyst stage has become a routine technique, occurring $90 \%$ of cases in 2010 (Figure 2).

Table 1 describes the 4 groups that were compared. Patients under 35 years of age, patients between 34 and 39 years of age, patients over 39 and patients who received oocytes from young donors (OD). The number of oocytes obtained at follicular aspiration, as expected, decreased with age. The proportion of cycles where ICSI was used as a method of insemination was similar in all groups (Table 1).

The number of embryos reaching the blastocyst stage was similar in the three groups of patients with a tendency to decrease according to age. However, when the oocyte belonged to a donor (OD), the blastocyst formation rate increased by more than $40 \%(\mathrm{p}<0.05)$. The rate was $40 \%$ among younger patients only $34 \%$ among older patients (Table 2). In contrast to our results, Thomas et al. (2010) reported that patient age patient age was negatively associated with the formation of blastocysts. Similarly, the percentage of cryopreserved embryos is higher among the OD group and patients under 35 than among patients over 35 years of age (Table 2).

The percentage of cycles with cancelled transfers was about $15 \%$ among patients over 34 years of age (Figure 3). Cancellations could be due to the low number of oocytes obtained during ovum pick-up, which leads to fewer or no embryos available, compared to pick-up with younger women. However, some reports have shown that blastocyst transfer may be applicable to any patient without compromising the probability of embryo transfer or pregnancy (Marek et al., 1999; Wilson et al., 2002).

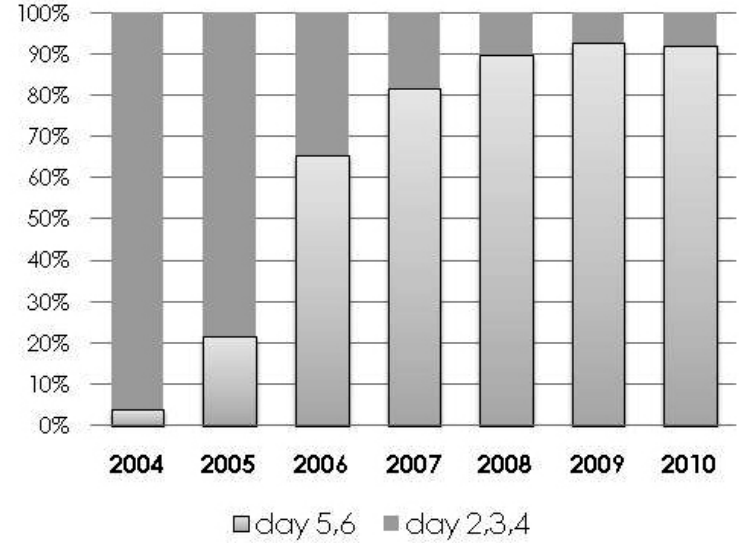

Figure 2: Proportion of transfers in days 2-4 versus 5-6. Oocyte donation cycles performed between 2004 ans 2010.

\section{TRANSFER CANCELLATION RATE ACCORDING TO THE FEMALE AGE}

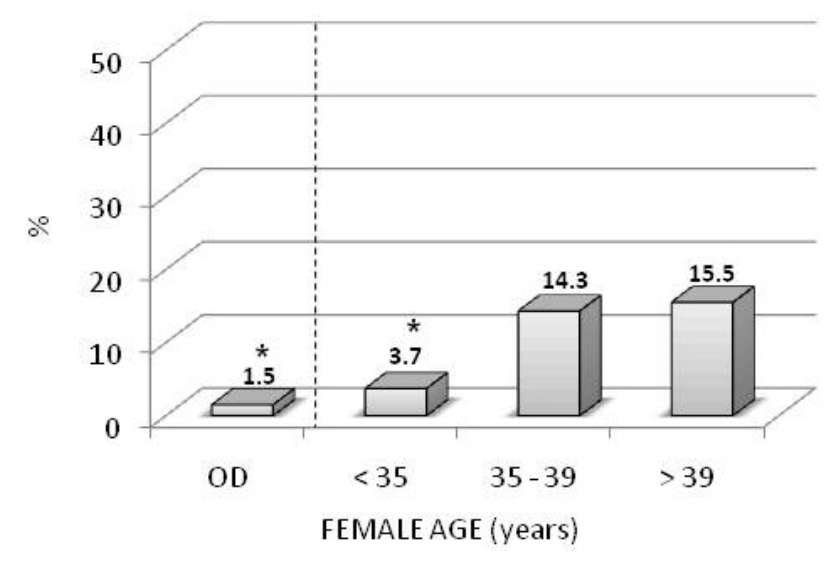

Figure 3: Transfer cancellation rate according to the female age $*$ : value is different to the other groups $(p<0.05)$

Table 1

Study Group

\begin{tabular}{|l|c|c|c|c|}
\cline { 2 - 5 } \multicolumn{1}{c|}{} & OD & $<35$ years & $35-39$ years & $>39$ years \\
\hline $\mathrm{N}^{\circ}$ of cycles & 1192 & 296 & 315 & 71 \\
\hline Donor age (years) & $24.61 \pm 3.13$ & & & \\
\hline Patient age (years) & $41.16 \pm 4.9$ & $31.28 \pm 2.36$ & $36.97 \pm 1.42$ & $41.0 \pm 1.36$ \\
\hline $\mathrm{N}^{\circ}$ of obtained oocytes & $9.81 \pm 3.49$ & $10.62 \pm 6.16$ & $8.01 \pm 4.69$ & $6.94 \pm 5.77$ \\
\hline ICSI cycles proportion & $39.85 \%$ & $44.93 \%$ & $44.44 \%$ & $43.66 \%$ \\
\hline
\end{tabular}

Numbers are mean \pm SD

*: the number of obtained oocytes is different between all groups $(p<0.001)$ 
Table 2

Blastocyst formation and cryopreservation rates

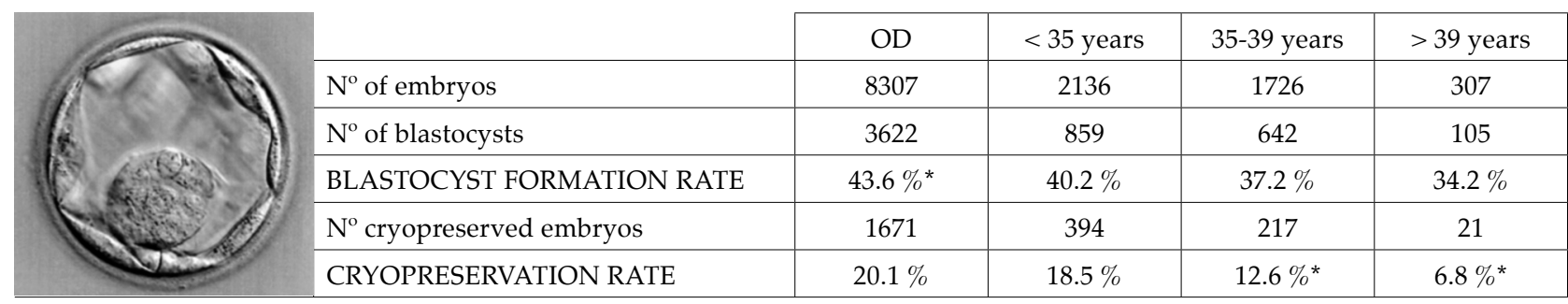

$*$ : Value is different to the other groups $(p<0.05)$

The pregnancy rate varied according to patient age: $48.4 \%$ for women under 35 and $38.6 \%$ for women over 39 (Figure 4). When cycles were done with donated oocytes from young woman, the pregnancy rate rose to $58.1 \%$, a value greater than in all groups of patients $(\mathrm{p}<0.005)$.

In this study, the maximum number of embryos transferred was two, which has been our policy since 2004 in order to avoid the risk of multiple pregnancies. The high rate of implantation shown in all the groups reflects the higher implantation potential of embryos transferred in the fifth day (Figure 4), also shown by other studies (Gardner et al., 1998; Blake et al., 2007). However, the implantation rate is lower in patients compared to the OD group. This can be explained by additional factors related to the cause of infertility in patients (Marino et al., 2011), since the egg donors are young women with no apparent reproductive problems. Moreover, there is evidence of severe changes in endometrial development in stimulated cycles (Haouzi et al., 2010). This may be beneficial for the recipients in the OD group because they were not stimulated.

Although the pregnancy rate for older patients is high with blastocyst transfer, the abortion rate is $59.1 \%$, which is significantly higher than the rates of the other groups (Figure 5).

This study, like others, has shown a decline in fertility for women over 35 years of age (Van Noord-Zaadstra et al., 1991; Perheentupa \& Huhtaniemi, 2099), which is significantly lower than the decline among women over 40 years of age (Marcus \& Brinsden, 1996). On the other hand, the lower probability of achieving a successful pregnancy related to increased maternal age is characterized by a higher prevalence of chromosomal abnormalities in the oocyte that lead ultimately to a significant reduction in implantation rates (Munné, 2002) and high rates of spontaneous abortion (Munné et al., 1995; Nybo-Andersen et al., 2000).

\section{CONCLUSIONS}

Extended embryo culture to the blastocyst stage can be implemented in an assisted reproduction program in order to increase the probability of pregnancy in patients, because natural selection can occur during embryo culture.

The potential of blastocyst implantation is high, allowing us to transfer fewer embryos and reduce the probability of multiple pregnancies. The blastocyst culture is an alternative that allows choosing one embryo to transfer in young women.

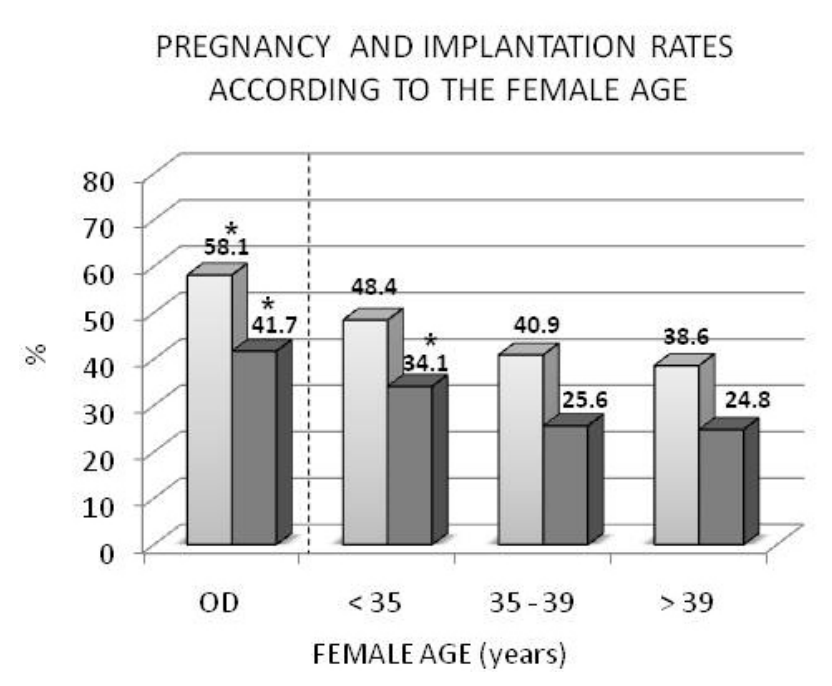

Figure 4: Pregnancy (light gray bars) and implantation (dark gray bars) rates according to the female age.

$*$ : value is different to the other groups $(p<0.05)$

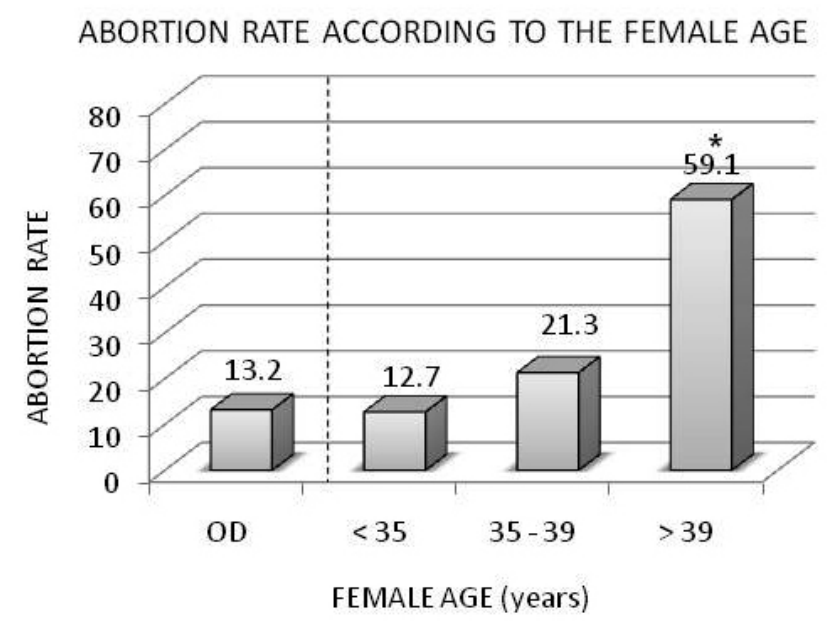

Figure 5: Abortion rate according to the female age. $*$ : value is different to the other groups $(p<0.05)$ 
The cancellation rate is higher among patients over 35 years of age, perhaps due to decreased ovarian reserve and the lower number of oocytes obtained, given that the embryos reach the blastocyst stage regardless of the patient's age.

\section{REFERENCES}

BARCROFT LC, OFFENBERG H, THOMSEN P, WATSON AJ (2003) Aquaporin proteins in murine trophectoderm mediate trans-epithelial water movements during cavitation. Dev Biol 256: 342-354.

BIGGERS JD, MCGINNIS LK, LAWITTS JA (2005) One-step versus two-step culture of mouse preimplantation embryos: is there a difference? Hum Reprod 20: 3376-3384.

BLAKE DA, FARQUHAR CM, JOHNSON N, PROCTOR M (2007) Cleavage stage versus blastocyst stage embryo transfer in assisted conception. Cochrane Database Syst Rev 4: CD002118.

BRAUDE P, BOLTON V, MOORE S (1988) Human gene expression first occurs between the four- and eight-cell stages of preimplantation development. Nature 332: 459-461.

GARDNER DK, LANE M (1997) Culture and selection of viable blastocysts: a feasible proposition for human IVF? Hum Reprod Update 3: 367-382.

GARDNER DK, LANE M (1998) Culture of viable human blastocysts in defined sequential serum-free media. Hum Reprod 3: 148-159.

GARDNER DK, LANE M, CALDERON I, LEETON J (1996) Environment of the preimplantation human embryo in vivo: metabolite analysis of oviduct and uterine fluids and metabolism of cumulus cells. Fertil Steril 65: 349-353.

GARDNER DK, SCHOOLCRAFT WB, WAGLEY L, SCHLENKER T, STEVENS J, HESLA J (1998) A prospective randomized trial of blastocyst culture and transfer in in-vitro fertilization. Hum Reprod 13: 3434-3440.

HAOUZI D, ASSOU S, DECHANET C, ANAHORY T, DECHAUD H, DE VOS J, HAMAMAH S (2010) Controlled ovarian hyperstimulation for in vitro fertilization alters endometrial receptivity in humans: protocol effects. Biol Reprod. 82:679-686.

MAREK D, LANGLEY M, GARDNER DK, CONFER N, DOODY KM, DOODY KJ (1999) Introduction of blastocyst culture and transfer for all patients in an in vitro fertilization program. Fertil Steril 72: 1035-1040.
MARCUS SF, BRINSDEN PR (1996) In-vitro fertilization and embryo transfer in women aged 40 years and over. Hum Reprod Update 2: 459468.

MARINO JL, MOORE VM, RUMBOLD AR, DAVIES MJ (2011) Fertility treatments and the young women who use them: an Australian cohort study. Hum Reprod 26: 473-479.

MUNNÉ S (2002) Preimplantation genetic diagnosis of numerical and structural chromosome abnormalities. Reprod Biomed Online 4: 183196.

MUNNÉ S, ALIKANI M, TOMKIN G, GRIFO J, COHEN J (1995) Embryo morphology, developmental rates, and maternal age are correlated with chromosome abnormalities. Fertil Steril 64: 382-391.

NYBO ANDERSEN AM, WOHLFAHRT J, CHRISTENS P, OLSEN J, MELBYE M (2000) Maternal age and fetal loss: population based register linkage study. BMJ 320: 1708-1712.

PAPANIKOLAOU EG, CAMUS M, FATEMI HM, TOURNAYE $H$ VERHEYEN G, VAN STEIRTEGHEM A, DEVROEY P (2006) Early pregnancy loss is significantly higher after day 3 single embryo transfer that after day 5 single blastocyst transfer in $\mathrm{GnRH}$ antagonist stimulated IVF cycles. Reprod Biomed Online 12: 60-65.

PERHEENTUPA A, HUHTANIEMI I (2009) Aging of the human ovary and testis. Mol Cell Endocrinol 299: 2-13.

SEPÚLVEDA S (2008) Fecundación y desarrollo en humanos In: LERNER J \& URBINA MT (eds) Reproducción Asistida. Caracas: Editorial Médica Panamericana. pp: 119-123.

SEPÚLVEDA S, GARCÍA J, ARRIAGA E, DÍAZ J, NORIEGA-PORTELLA L, NORIEGA-HOCES L. (2009) In vitro development and pregnancy outcomes for human embryos cultured in either a single medium or in a sequential media system. Fertil Steril 91:1765-1770.

THOMAS MR, SPARKS AE, RYAN GL, VAN VOORHIS BJ (2010) Clinical predictors of human blastocyst formation and pregnancy after extended embryo culture and transfer. Fertil Steril 94: 543-548.

VAN NOORD-ZAADSTRA BM, LOOMAN CW, ALSBACH H, HABBEMA JD, TE VELDE ER, KARBAAT J (1991) Delaying of childbearing: effect of age on fecundity and outcome of pregnancy. BMJ 302:1361-1365.

WILSON M, HARTKE K, KIEHL M, RODGERS J, BRABEC C, LYLES R (2002) Integration of blastocyst transfer for all patients. Fertil Steril 77: 693-696. 
